\title{
Estudo interinstitucional das atitudes sociais em relação à inclusão no ensino superior
}

\section{Interinstitutional study of social attitudes in relation to the inclusion in college}

\author{
Cristiane R. X. Fonseca-Janes*, Sadao Omote** \\ *Grupo de Pesquisa Diferença, Desvio e Estigma, **Departamento de Educação Especial Faculdade de Filosofia e \\ Ciências/UNESP Programa de Pós-Graduação em Educação
}

\begin{abstract}
Resumo
O objetivo deste trabalho foi comparar as atitudes sociais em relação à inclusão, mantidas por estudantes de três instituições de ensino superior brasileiro. Participaram desta pesquisa 74 estudantes concluintes de cursos de formação de professores. Os dados foram coletados por meio da Escala Likert de Atitudes Sociais em relação à Inclusão. Os escores das atitudes sociais desses participantes foram comparados por meio da prova de Kruskal-Wallis e verificamos que existe diferença significante $(p<0.002)$ entre os participantes dos cursos. Esse resultado é um indicativo de que a formação nestes cursos possui suas particularidades, sejam elas regionais ou resultantes da estruturação curricular.
\end{abstract}

Palavras chave: 1. Educação Especial. 2. Atitudes Sociais. 3. Educação Inclusiva. 4. Estudantes Universitários.

\section{Introdução}

A Educação Especial brasileira, pensada na perspectiva da educação inclusiva, é uma área específica do conhecimento que só tem a contribuir para a efetivação de uma sociedade inclusiva, uma vez que tem conhecimentos acumulados sobre o ensino de pessoas com deficiência, que poderá beneficiar toda a comunidade.

Gardou (2011), ao discutir a deficiência numa perspectiva inclusiva, afirma que somente desconstruindo conceitos cristalizados, próprios do obscurantismo, podemos atingir o ideal de "uma sociedade menos excludente, menos normativa, sem masmorras nem grades" (p. 22). Para esse autor, uma sociedade inclusiva, é uma sociedade pensada para todos sem pertencimentos a grupos particulares, é garantir a singularidade de cada ser dentro dos ideais universais, é garantir qualidade de vida e acesso a todos. Nessa sociedade com qualidade e acesso o resultado é a educação inclusiva.

A educação inclusiva é uma educação de qualidade direcionada a todos os alunos da comunidade escolar. Admite-se que, ao conviver com a diversidade, todos os integrantes da comunidade escolar têm mais benefícios do que perdas, desde que estejam amparados por recursos materiais e humanos de qualidade. Com um sistema educacional munido de recursos qualificados, as pessoas com deficiência ou diferenças expressivas não são apenas inseridas nas escolas, mas fazem parte de uma comunidade escolar que prima pela aprendizagem significativa. Para a implementação da educação inclusiva, são necessários administradores preocupados com a reforma, com a reestruturação e com a renovação de suas unidades de ensino. Cabe ainda a esta unidade, a formação continuada do seu corpo docente em metodologias de ensino que privilegiem uma abordagem de ensino progressista (Mittler, 2003) e que incluam estratégias para a mudança de atitudes sociais, bem como recursos materiais e outros profissionais de apoio. Com esses passos, podemos estar próximos de uma educação inclusiva, cujo resultado pode ser um sistema educacional fortalecido e eficiente no quais todas as crianças teriam acesso e permanência garantida, com a oferta de oportunidades que lhes assegurem um aproveitamento máximo, conforme as condições de cada aluno. Salienta-se que essa perspectiva não difere das metas traçadas por todos aqueles que visam a uma educação democrática de qualidade, gratuita e laica, desde o Manifesto dos Pioneiros de 1932 ou mesmo o Manifesto dos Educadores Mais Uma Vez Convocados de $1959^{1}$.

\footnotetext{
${ }^{1}$ O Manifesto dos Pioneiros da Educação Nova de 1932 foi um documento que partiu de um grupo de 22 jovens intelectuais que procuram levantar idéias pedagógicas da história e da filosofia da educação brasileira. O redator do Manifesto foi Fernando Azevedo, cujos ideais educacionais pautavam-se nas idéias de John Dewey e de Émile Durkhein. Nesse documento existem discussões teórica, filosófica, histórica, política e formulações pedagógicas didáticas sobre a educação. Para seus signatários as reformas econômicas não deveriam estas dissociadas das reformas educacionais. Os problemas educacionais eram advindos da falta de uma filosofia da educação e da falta de uma visão científica dos problemas educacionais. Para eles a Filosofia da educação deveria adequar a escola à modernidade. Eles se posicionavam contra uma educação dual e uma escola tradicionalista. Defendiam a escola socializada, a Educação como dever do Estado, uma escola comum, única para todos, laica, gratuita, obrigatória, co-
} 
Para concretude de uma comunidade escolar inclusiva precisamos de soluções didático-pedagógicas e de medidas avaliativas solidamente fundamentadas para a verificação dos processos de inclusão escolar.

Uma alternativa de avaliação da educação inclusiva pode envolver a questão das atitudes sociais em relação à inclusão. Uma pessoa, em interação com o ambiente social, forma impressões sobre as outras pessoas e essas impressões direcionam o seu comportamento. As atitudes são formadas como conseqüência da ação direta da pessoa com a tomada de conhecimento do meio ambiente. Assim, podemos dizer que nossas atitudes são formadas durante nosso processo de socialização.

Para Rodrigues, Assmar \& Jablonski (2007, p. 97-98), os elementos característicos das atitudes sociais são “[...] a) uma organização duradoura de crenças e cognições em geral; b) uma carga afetiva pró ou contra; c) uma predisposição à ação; d) uma direção a um objeto social". Assim, nós conceituamos atitudes sociais em relação à inclusão como uma reorganização ${ }^{2} \mathrm{de}$ crenças, cognições, percepções e conceituações dotadas de afetividade contra ou a favor da inclusão de grupos minoritários, esta poderá direcionar ações coerentes ou não com os juízos de valor de um ser cognitivo relativos à inclusão social de grupos minoritários.

A característica básica das atitudes sociais em relação à Inclusão é que não podemos observá-la, mas podemos inferir se uma pessoa é favorável à inclusão de grupos minoritários por meio de inferências de componentes observáveis, tais como: o componente cognitivo, o componente afetivo e o componente comportamental.

Atitudes sociais favoráveis em relação à inclusão podem ser construídas por meio de intervenção, uma das possibilidades é a criação de estratégias para que os grupos minoritários possam demonstrar suas potencialidades na aquisição do conhecimento acumulado pela humanidade.

Nas discussões atuais acerca da educação inclusiva, tem sido dada especial ênfase às atitudes sociais de toda a comunidade escolar e principalmente às do professor. A partir da compreensão das atitudes sociais dos professores em relação à inclusão, é possível ter alguma idéia das condutas que eles adotam em suas salas de aula. Um professor com atitudes sociais desfavoráveis em relação à inclusão dificilmente consegue enfrentar o desafio de promover ensino de qualidade para os alunos

educação dos sexos. Os signatários do Manifesto dos Pioneiros foram os seguintes personagens históricos: Fernando Azevedo, Roldão Lopes de Barros, Hermes Lima, Paschoal Lemme, Edgard Sussekind de Mendonça, Júlio de Mesquita Filho, Armanda Álvaro Alberto, Raul Briquet, Mário Casassata, Atílio Vivacqua, Francisco Venancio Filho, Afrânio Peixoto, Antônio Sampaio Dória, Roquete Pinto, Frota Pessoa, Noemy Silveira, Garcia de Resende, Almeida Júnior, J. P. Fontanelle, Paulo Maranhão, Cecília Meirelles, Nóbrega Da Cunha, Raul Rodrigues, Anísio Teixeira e Lourenço Filho. (Azevedo, 1932; 1959).

2 Optamos pelo termo "reorganização", uma vez que a expressão "Atitudes Sociais" é considerada uma organização, e aqui teorizamos o conceito de Atitudes Sociais em Relação à Inclusão, que é um estudo epistemológico do conceito de Atitudes Sociais, transcendendo ao próprio conceito, pensando aqui nos aspectos metafísicos (Zingano, 2003). Consequentemente, estamos pensando nas estruturas subjacentes que podem mudar um conceito já estabelecido socialmente. que apresentam diferenças expressivas em relação ao aluno comum. Bender, Scott \& Vail (1995) evidenciaram que os professores do ensino comum com atitudes negativas em relação à inclusão utilizavam estratégias de ensino inclusivas menos frequentemente que os professores com atitudes positivas.

As atitudes sociais, assim como outras variáveis não imediatamente visíveis, tais como as crenças, os valores e as concepções de ensino e aprendizagem, podem determinar a qualidade da relação interpessoal entre o professor e o aluno, podendo comprometer o processo inclusivo de ensino e aprendizagem. Destaque-se que:

[...] os professores são agentes importantes na construção da educação inclusiva, pois depende essencialmente deles a criação de um clima acolhedor para todos os alunos na sala de aula. As suas ações docentes e sociais na sala de aula se constituem como elementos críticos para o ensino inclusivo. Para tanto, além da capacitação didático-pedagógica para lidar com a diversidade de características e necessidades educacionais de seus alunos, precisam desenvolver atitudes genuinamente favoráveis em relação aos princípios e práticas da inclusão. (Omote, 2003, p.1)

Como parte da preocupação em implementar a educação inclusiva, as atitudes sociais dos professores em relação à inclusão vêm sendo investigadas nos últimos anos no Grupo de Pesquisa: Diferença, Desvio e Estigma. O gênero, a idade cronológica, o tempo de experiência docente no ensino comum, o nível de escolaridade e a experiência no ensino do aluno com deficiência são algumas das variáveis do professor, que podem estar criticamente relacionadas às suas atitudes sociais em relação à inclusão, embora seus efeitos específicos não estejam cabalmente esclarecidos.

Foi evidenciado que os professores da Educação Infantil e os estudantes do CEFAM e de Pedagogia apresentavam atitudes sociais em relação à inclusão mais favoráveis que os professores do Ensino Fundamental, Ciclos I e II, e os do Ensino Médio (Omote et. al., 2003; 2005). Os autores aventaram uma possível explicação baseada na relação desses estudantes e professores com a situação de uma classe da qual participa algum aluno com deficiência.

Os estudantes do CEFAM e de Pedagogia não enfrentavam situações concretas de lidar com a presença de aluno com deficiência em sala de aula, razão pela qual a adesão à posição considerada politicamente correta poderia ter ocorrido com maior facilidade. Os professores da Educação Infantil também poderiam demonstrar atitudes amplamente favoráveis à inclusão, em vista da sua situação profissional de lidar com classes pouco numerosas e com atividades que não colocavam especial exigência em termos de aprendizagem escolar. Já os professores do Ensino Fundamental e do Ensino Médio enfrentavam situações de tal natureza na sala de aula - classe numerosa e atividades escolares que exigiam demonstração de competência intelectual - que poderiam mais facilmente visualizar a dificuldade que enfrentariam com a presença de algum aluno com necessidades especiais, como aquele que não se comunica oralmente ou aquele que não enxerga. 
Embora existam resultados contraditórios, de modo geral pode-se sugerir que as atitudes sociais positivas em relação à inclusão são apresentadas por professores do gênero feminino, mais jovens, com experiência docente no ensino comum, com menos tempo de experiência docente no ensino comum, com nível mais baixo de escolaridade, com experiência no ensino de alunos deficientes, professores com mais tempo de experiência com alunos deficientes, tipo de deficiência e grau de comprometimento, autopercepção da competência, preparação profissional, formação e competência docente (Fonseca-Janes, 2010; FonsecaJanes; Omote, 2013; Omote, Fonseca-Janes \& Vieira, 2014).

Ressaltamos que embora alguns estudos apontem que pessoas do gênero feminino tendam a ter atitudes sociais mais favoráveis em relação à inclusão, entretanto os estudos de Patrick (1987), Rizzo \& Vispoel (1991), Rizzo \& Wright (1988) indicam que não existe essa diferença de gênero nas atitudes de professores no ensino de pessoas com deficiência ou de estudantes (Fonseca-Janes, 2010).

No Brasil, há carência de estudos de campo acerca das atitudes sociais em relação à inclusão. Quando, em algum estudo, o autor se refere às atitudes em relação à inclusão, frequentemente $\mathrm{o}$ faz de modo vago e superficial, a partir do que pode ser inferido grosseiramente nos relatos verbais de seus entrevistados, talvez por não haver tradição de se utilizarem instrumentos padronizados de mensuração de fenômenos dessa natureza.

Nas várias discussões acerca da inclusão, tem sido constantemente lembrada a necessidade de uma profunda mudança nas atitudes por parte de todas as pessoas envolvidas. Diferentemente do modelo de integração, no qual a ênfase recaía sobre a capacitação do próprio deficiente para fazer face às demandas do meio ao qual viria a ser integrado, na nova perspectiva de trabalho, é de extrema importância a compreensão das atitudes de todas as pessoas envolvidas na educação que pretende ser inclusiva em especial, a de futuros profissionais especializados em Educação Especial, que atuarão diretamente a efetivação de processos inclusivos de pessoas com deficiência.

Desta forma, este trabalho teve por objetivo verificar se nos quatro modelos de formação de recursos humanos em Educação Especial brasileiro apresentam diferenças significativas nas atitudes sociais em relação à inclusão de estudantes dos últimos semestres dos cursos de Pedagogia da Faculdade de Filosofia e Ciências da Universidade Estadual Paulista "Júlio de Mesquita Filho" - UNESP, Campus de Marília, SP, BR, com aprofundamento em Educação Especial, de Licenciatura em Educação Especial da Universidade Federal de Santa Maria - UFSM, RS, BR e de Licenciatura em Educação Especial da Universidade Federal de São Carlos, SP, BR.

\section{Método}

\section{Participantes}

Participaram desta pesquisa estudantes concluintes de quatro cursos de formação de professores especializados para o ensino de pessoas com deficiências de ambos os gêneros, em um total de 74 participantes, que foram distribuídos em quatro grupos:

- Grupo 1: 11 estudantes do Aprofundamento em Educação Especial, último semestre do curso de Pedagogia da Faculdade de Filosofia e Ciências da Universidade Estadual Paulista "Júlio de Mesquita Filho" - UNESP; com idade mínima de 21 anos e máxima de 32 anos, sendo a média de idade de 24,2 anos e desvio padrão de 4,02.

- Grupo 2: 20 estudantes do sétimo semestre do curso de Licenciatura em Educação Especial da Universidade Federal de São Carlos - UFSCar; com idade mínima de 20 anos e máxima de 43 anos, sendo a média de idade de 25 anos e desvio padrão de 6,42 .

- Grupo 3: 33 estudantes do oitavo semestre, último semestre, do curso de Licenciatura em Educação Especial da Universidade Federal de Santa Maria - UFSM, período diurno; com idade mínima de 20 anos e máxima de 49 anos, sendo a média de idade de 23,8 anos e desvio padrão de 5,39 .

- Grupo 4: 10 estudantes do oitavo semestre do curso de Licenciatura em Educação Especial da Universidade Federal de Santa Maria - UFSM, período noturno; com idade mínima de 21 anos e máxima de 32 anos, sendo a média de idade de 26 , 2 anos e desvio padrão de 3,22.

\section{Material}

Utilizamos a primeira versão da Escala Likert de Atitudes Sociais em relação à Inclusão (ELASI), que é um instrumento construído e validado pelo grupo de pesquisa Diferença, Desvio e Estigma (Omote, 2005), para a mensuração de atitudes sociais em relação à inclusão. Esse instrumento possui 35 itens, cada um constituído por um enunciado seguido de 05 alternativas que indicam a extensão em que o respondente concorda com o seu conteúdo ou dele discorda. As alternativas para a resposta são: (a) concordo inteiramente, (b) concordo mais ou menos, (c) nem concordo nem discordo, (d) discordo mais ou menos, e (e) discordo inteiramente. Desses 35 itens, 30 medem as atitudes sociais e cinco correspondem à escala de mentira. Dos itens relativos às atitudes sociais, 15 apresentam enunciados positivos, isto é, a concordância com o seu conteúdo corresponde à expressão de atitudes sociais favoráveis à inclusão; outros 15 itens apresentam enunciados negativos, isto é, a concordância com o seu conteúdo corresponde à expressão de atitudes sociais desfavoráveis à inclusão. A escala de mentira foi 
incluída na ELASI, como um dispositivo para obter indícios da confiabilidade das respostas fornecidas pelos participantes, já que, conforme estudos prévios, as respostas a esses 05 itens são inteiramente previsíveis.

\section{Procedimentos}

Procedimento de coleta de dados.

Após autorização das três universidades envolvidas na pesquisa agendamos as coletas de acordo com a disponibilidade e concordância dos coordenadores e estudantes para aplicar a ELASI aos estudantes concluintes dos cursos.

\section{Formas de tratamento de dados.}

Os dados coletados por meio da ELASI foram armazenados em um banco de dados específico, criado pelo grupo de pesquisa Diferença, Desvio e Estigma. Cada uma das respostas dos participantes recebe uma codificação específica. $\mathrm{Na}$ análise de dados, os códigos são substituídos por notas para o cálculo de escores individuais de atitudes sociais. Para os itens positivos, é atribuída a nota 5 à alternativa (a), de concordância plena, 4 à alternativa (b) e assim por diante até a nota 1 à alternativa (e). Para os itens negativos, o sentido de atribuição de notas é invertido, já que, diante deles, a concordância plena com o conteúdo expressa as atitudes mais desfavoráveis. O escore total de cada participante é obtido pela soma das notas atribuídas a 30 itens que medem atitudes sociais e pode variar de 30 a 150 , sendo que quanto maior for o escore mais favoráveis são as atitudes sociais em relação à inclusão.

A escala de mentira recebe outra pontuação: se a resposta fornecida a um item for aquela que é esperada, era atribuída a nota 0 e, caso contrário, o item recebia a nota 1. Assim, os escores da escala de mentira podem variar de 0 a 5 , sendo que o valor 0 ou próximo dele indica maior confiabilidade dos dados.

Os escores das atitudes sociais em relação à inclusão dos participantes, obtidos por meio da ELASI, foram transferidos para uma planilha com todos os demais dados dos participantes para que possamos realizar comparações interinstitucionais, comparações intrainstitucionais, e comparações entre o estudo desenvolvido em 2010 (Fonseca-Janes, 2010).

\section{Formas de análise de dados}

Preliminarmente, analisamos as respostas aos itens da escala de mentira. Em todos os estudantes dos quatro cursos os escores podem variar de zero a cinco. Esse mecanismo é usado para verificar se os participantes de um modo geral responderam ao instrumento com a devida seriedade para que os dados coletados tenham a confiabilidade necessária.

Verificamos os resultados dos escores obtidos por meio da ELASI. Os escores foram distribuídos em tabelas com a indicação das medidas de variação, representada pelo menor e maior escore, a medida de tendência central, representada pela mediana, e a medida de dispersão, representada pelo quartil 1 e quartil 3.

Esses dados ainda foram submetidos aos testes estatísticos para verificar se existe diferença entre os resultados entre os três grupos por meio da prova de Kruskal-Wallis.

\section{Resultados e discussão das atitudes sociais em relação à inclusão}

A tabela 1 apresenta os parâmetros relativos aos escores da ELASI obtidos por participantes no início do processo de formação e no último ano do curso. São apresentadas a medida de variação, representada pelo menor e maior escore, a medida de tendência central, representada pela mediana, e a medida de dispersão, representada pelo quartil 1 e quartil 3 .

Tabela 1- Síntese de escores da ELASI obtidos por participantes dos cursos de formação de Recursos Humanos em Educação Especial

\begin{tabular}{l|c|c|c} 
IES & $\begin{array}{c}\text { Variação } \\
\text { (Min.-Máx.) }\end{array}$ & Mediana & $\begin{array}{c}\text { Dispersão } \\
\text { (Q1 - Q3) }\end{array}$ \\
\hline UNESP & $132-146$ & 140 & $138-141$ \\
UFSCar & $129-147$ & 141,5 & $138,75-144,25$ \\
UFSM diurno & $108-150$ & 131 & $127-139$ \\
UFSM noturno & $103-150$ & 131,5 & $125,5-143$ \\
\hline
\end{tabular}

Os escores desses quatro grupos de participantes foram comparados por meio da prova de Kruskal-Wallis e verificamos que existe diferença estatisticamente significante $(\mathrm{p}<0.002)$ entre os participantes dos cursos. Esse resultado é um indicativo de que formação nestes cursos possui suas particularidades, sejam elas regionais ou resultantes da estruturação curricular.

Como o resultado do teste demonstra existir uma diferença estatisticamente significante entre os grupos, achamos por bem não agrupá-los em um único grupo e respeitar a unicidade de cada uma das unidades de ensino. Para demonstrar as diferenças existentes nas atitudes sociais em relação à inclusão dos participantes desta pesquisa, no quadro 1 poderemos verificar as diferenças existentes das atitudes por meio das comparações 2 a 2 realizadas por meio da comparação múltipla de Dunn.

\begin{tabular}{|c|c|}
\hline $\begin{array}{c}\text { Comparação dos escores dos participantes } \\
\text { nas Unidades Ensino Superior }\end{array}$ & Valor de p \\
\hline \hline UNESP versus UFSCar & $\mathrm{p}>0,05$ \\
UNESP versus UFSM_Diurno & $\mathrm{p}>0,05$ \\
UNESP versus UFSM_Noturno & $\mathrm{p}>0,05$ \\
UFSCar versus UFSM_Diurno & $\mathbf{p}<\mathbf{0 , 0 1}$ \\
UFSCar versus UFSM_Noturno & $\mathrm{p}>0,05$ \\
UFSM_Diurno versus UFSM_Noturno & $\mathrm{p}>0,05$ \\
\hline
\end{tabular}

Quadro 1 - Distribuição das comparações 2 a 2 dos escores obtidos na ELASI dos participantes

Com os dados demonstrados no quadro 1, é possível verificar que os participantes da Licenciatura em Educação Especial, diurno, da Universidade Federal de Santa Maria são os que se diferenciam de todos os demais participantes, uma vez que apresentam 
diferenças estatisticamente significantes dos demais participantes. Assim, nesse contexto específico e em comparação com as demais unidades de Ensino Superior, estes participantes apresentam atitudes sociais em relação à inclusão menos favoráveis. Talvez, isto se justifique pelo fato destes participantes terem uma formação crítica com relação à Educação Inclusiva (Possas, 2014).

Hastings e Oakford (2003) sugerem em seus estudos sobre as atitudes sociais de estudantes e professores com relação à inclusão de crianças com deficiência, que as atitudes não são o único fator para a determinação do sucesso de programas que visem a inclusão. Para eles, as atitudes contribuem significativamente para a efetivação, mas também são necessários suporte e recursos apropriados para o professor, e a crítica a falta de suporte necessário ao professor e as crianças com deficiência nas salas regulares é um dos motivos que pode ter levado às atitudes sociais menos favoráveis dos participantes do curso de licenciatura em Educação Especial diurno da UFSM.

O resultado desse trabalho é de extrema valia, uma vez que até o momento não existem pesquisas interinstitucionais sobre atitudes sociais em relação à inclusão. Tínhamos como hipótese, que a formação condensada na área do Aprofundamento em Educação Especial poderia ser uma das possibilidades para que as atitudes sociais em relação à inclusão fossem menos favoráveis e isto não se procedeu, talvez o instrumento de coleta de dados deva ser revisto e outras replicações se fazem necessárias para a confirmação e maiores sobre a formação do professor especialista e do professor generalista.

Uma pessoa em interação com o ambiente social forma impressões sobre as outras pessoas, e essas impressões direcionam o seu comportamento. As atitudes são formadas como conseqüência da ação da pessoa com a tomada de conhecimento do meio ambiente. As atitudes são formadas durante nosso processo de socialização e isto ocorre em vários espaços sociais, sendo os cursos estudados espaços para a efetivação desse processo social.

As atitudes são formadas por meio da experiência e da aprendizagem. Uma pessoa tende a formar atitudes em relação aos agentes situacionais quanto mais expostas forem à experiências diretas. $\mathrm{O}$ fato dos participantes terem disciplinas que ofereceu contato com a temática da inclusão e terem tido contato com pessoas com deficiência nos estágios supervisionados na sala de aula regular também podem ser alguns fatores para mudança de suas atitudes em relação à inclusão. A variável competência docente (Rizzo \& Wright, 1988) também pode ser um dos motivos para todos os participantes já terem atitudes mais favoráveis do que a de estudantes de outros cursos, uma vez que os participantes estão em fase de conclusão de curso de graduação.

As atitudes sociais favoráveis à inclusão são uma das condições para construção de uma sociedade inclusiva, uma vez que podem organizar ou reorganizar crenças e cognições sobre as diferenças, direcionar a afetividade de modo a ser favorável ou desfavorável com relação às diferenças e, principalmente, direcionar a ação para a aceitação ou negação das diferenças. A mudança de atitudes em relação à inclusão destes estudantes e futuros educadores pode criar técnicas e recursos que auxiliam todos os alunos.

Conforme apontamos, inicialmente, muitas outras variáveis podem modificar as atitudes sociais, e algumas dessas estão presentes nos participantes destes cursos, como é o caso da incidencia de 71 dos participantes serem do gênero feminino (Pearman et al, 1992).

\section{Conclusão}

A efetivação dos ideais da educação inclusiva pode ocorrer por meio da construção de atitudes sociais favoráveis à inclusão, uma vez que estas podem organizar ou re-organizar crenças e cognições sobre as diferenças, direcionar a afetividade de modo a ser favorável com relação às diferenças e, principalmente, direcionar a ação para a aceitação das diferenças. Assim, as atitudes sociais se constituem como bons preditores das ações que são direcionadas ao objeto atitudinal.

O presente estudo evidencia que mesmo com um dos grupos estudados tenham atitudes menos favoráveis do que os demais, todos possuem os escores acima de 100, o que já pode ser considerado atitudes sociais mais favoráveis em relação à inclusão do que em outros cursos. O resultado sugere que estas atitudes sociais mais favoráveis podem ser decorrentes da formação específica na área da Educação Especial, convêm à replicação dos estudos em outros cursos para a confirmação da hipótese.

Estudos anteriores apontam que o processo formativo pode ser uma das variáveis para modificar atitudes sociais em relação à inclusão, neste estudo específico os participantes tiveram no processo de formação universitária matrizes curriculares estruturadas para cursos voltados ao atendimento, acolhimento e processo de ensino e aprendizagem voltados à diversidade.

É sabido que as atitudes se formam por meio da experiência e da aprendizagem. Assim, no que se referem às atitudes sociais os cursos na área específica, seja em licenciatura ou em aprofundamento, formam egressos com potencialidades para a efetivação dos ideais da Educação Inclusiva.

Inúmeros estudos apontam a existência de resultados conflitantes entre as literaturas internacionais e nacionais nos estudos das atitudes sociais. Mas, pensamos ser os conflitos existentes em um fenômeno o que o torna justificável para futuros estudos, uma vez que as ciências, assim como a filosofia, se fundamentam nos estudos dos conflitos dos fenômenos. Estes resultados fortalecem a necessidade de replicações e mais estudos sobre essa variável.

\section{Referências}

Azevedo, F. (1932). A reconstrução educacional no Brasil. Ao povo e ao governo. O manifesto dos pioneiros da educação nova. São Paulo: Ed. Nacional. Azevedo, F. (1959). Manifesto dos Educadores: mais 
uma vez convocados. In Revista HISTEDBR On-line, Campinas, n. especial, 205-220, ago 2006.

Fonseca-Janes, C.R.X. (2012). Modelo para mudanças de atitudes sociais em relação à inclusão. Revista Galego-Portuguesa de Psicoloxía e Educación, 20(1), p.83-98. http://ruc.udc.es/bitstream/2183/12110/1/ RGP_20_2012_art 6.pdf

Fonseca-Janes, C.R.X. (2010). A formação dos estudantes de pedagogia para a Educação Inclusiva: estudo das atitudes sociais e do currículo. Tese de Doutorado, Faculdade de Filosofia e Ciências, Universidade Estadual Paulista, Marília, São Paulo, Brasil.

http://www.dominiopublico.gov.br/pesquisa/DetalheO braForm.do? select_action $=\&$ co_obra $=200909$

Fonseca-Janes, C.R.X. \& Omote,S. (2013). Atitudes sociais em relação à inclusão: o curso de Pedagogia da Faculdade de Ciências e Tecnologia da UNESP. Nuances: estudos sobre Educação, Presidente Prudente, SP, 24 (2), p. 158-173. http://dx.doi.org/10.14572/nuances.v24i2.2486

GARDOU, C. (2011). Pensar a deficiência numa perspectiva inclusiva. Revista Lusófona de Educação, 19(1), p. 13-23.

Hastings, R.P. \& Oakford, S. (2003). Student teachers' attitudes toward the inclusion of children with special needs. Educational Psychology, 23(1), 87-94.

Mittler, P. (2003). Educação inclusiva: Contextos sociais. Porto Alegre: Artmed.

Omote, S. (2003). O uso de $\chi^{2}$ na análise de itens para construção de uma escala de atitudes sociais em relação à inclusão. In: V Simpósio em Filosofia e Ciência, Trabalho e conhecimento: desafios e responsabilidades das ciências. Anais eletrônicos da V Simpósio em Filosofia e Ciência, Trabalho e conhecimento: desafios e responsabilidades das ciências (p. 01-09). Marília: SAEPE.

Omote, S. (2005). A construção de uma escala de atitudes sociais em relação à inclusão: notas preliminares. Revista Brasileira de Educação Especial, 11(1), 33-47.

Omote, S. ; Fonseca-Janes, C. R. X. \& Vieira, C. M. (2014). Variables personales Del profesor y sus relaciones em grupo. En. Omote, S.; Braga, T.M.S.; Chacón, M. C. M \& Montalvo, D. (Eds.), Reflexiones internacionales sobre la formación de profesores para la atención a los alumnos con necesidades educativas especiales. (pp. 149-178). Alcalá de Henares (España) : UAH.

Omote, S.; Oliveira, A. A. S.; Baleotti, L. R. \& Martins, S. E. S. O. (2003). Mudança de atitudes sociais de alunas do CEFAM em relação à inclusão. In: V SIMPÓSIO EM FILOSOFIA E CIÊNCIA, Marília: UNESP Marília Publicações.

Patrick, G. Improving attitudes toward disabled persons. Adapted Physical Activity Quarterly, v. 4, p. 316-325, 1987.

Pearman, E. L., Huang, A. M., Barnhart, M. W. \& Melblom, C. (1992). Educating all students in school: attitudes and beliefs about inclusion. Education and Training in Mental Retardation, 27, 176-182.

Rizzo, T. L., \& Vispoel, W. P. (1991). Physical educators' attributes and attitudes toward teaching students with handicaps. Adapted Physical Activity Quarterly, 8(1), 4-11.

Rizzo, T.L., \& Wright, R. G. (1988). Selected attributes related to physical educators' attitudes toward teaching students with handicaps. Mental Retardation, 26, 307-309.

Rodrigues, A., Assmar, E. M. L.\& Jablonsky, B. (2007). Psicologia social. 25. ed. Petrópolis, Rio de Janeiro: Vozes.

Zingano, M. (2003). Forma, Matéria e Definição na Metafísica de Aristóteles. Cad. Hist. Fil. Ci., Campinas, 13 (2), p. 277-299. 